\title{
CONJUGALIDADE E PARENTALIDADE SUBSEQUENTES À PERDA GESTACIONAL: REVISÃO SISTEMÁTICA
}

\author{
Gabriela Vescovi \\ Universidade Federal do Rio Grande do Sul, Porto Alegre-RS, Brasil \\ Flávia Santos da Silva \\ Daniela Centenaro Levandowski \\ Universidade Federal de Ciências da Saúde de Porto Alegre, Porto Alegre-RS, Brasil \\ Crístofer Batista da Costa \\ Faculdade do Centro de Estudos da Família e do Indivíduo, Porto Alegre-RS, Brasil
}

\begin{abstract}
RESUMO
O objetivo deste estudo foi analisar a literatura científica no período de janeiro de 2006 a março de 2021 sobre as repercussões da perda gestacional na conjugalidade e na parentalidade. Trata-se de uma revisão sistemática que envolveu consulta às bases Pubmed, PsycNET e Portal BVS, por meio de descritores estabelecidos previamente, resultando em 16 estudos elegíveis para análise. Foram encontrados resultados contrastantes quanto às repercussões da perda gestacional na parentalidade, sugerindo a necessidade de investigação de fatores de proteção. A perda apresentou-se como risco à conjugalidade, porém a comunicação conjugal emergiu como fator de proteção que merece maior investigação. Estudos futuros devem adotar métodos qualitativos ou mistos, incluir a perspectiva masculina sobre o tema e avaliar intervenções conjugais e familiares.
\end{abstract}

Palavras-chave: Aborto espontâneo; Casamento; Relações pais-criança.

\section{CONJUGALITY AND PARENTHOOD AFTER PREGNANCY LOSS: SYSTEMATIC REVIEW}

\section{AbSTRACT}

This study aimed to analyze the scientific production from Jan 2006 to Mar 2021 regarding the repercussions of pregnancy loss on conjugality and parenthood. It is a systematic review carried out in Pubmed, PsycNET, and Portal BVS database through previously established descriptors, resulting in 16 eligible studies. We found contrasting results regarding repercussions in parenthood, suggesting the need to investigate protective factors also. Pregnancy loss shows as a risk for marital relationships, but marital communication emerged as a possible protection factor that deserves further investigation. Future research should adopt qualitative and mixed methods, include men's perspectives, and evaluate marital and family interventions.

Keywords: Spontaneous abortion; Marriage; Parent-child relations. 


\section{CONYUGALIDAD Y PARENTALIDAD SUBSECUENTE A PÉRDIDA GESTACIONAL: REVISIÓN SISTEMÁTICA}

\section{RESUMEN}

El objetivo de este estudio ha sido analizar la literatura científica en el período de enero de 2006 hasta marco de 2021 sobre las repercusiones de la pérdida gestacional en la conyugalidad y en la parentalidad. Esta es una revisión sistemática realizada en las bases Pubmed, PsycNET y Portal BVS, con los descriptores establecidos previamente, resultando en 16 estudios elegibles. Se encontraron resultados contrastantes en cuanto a las repercusiones en la crianza de los hijos, sugiriendo la necesidad de investigar también los factores protectores. La pérdida se presentó como un riesgo para la conyugalidad, sin embargo, la comunicación marital emergió como un posible factor protector que merece más estudios. Se indica que futuras investigaciones adopten métodos cualitativos, que incluyan la perspectiva masculina y evalúen intervenciones conyugales y familiares.

Palabras clave: Aborto espontáneo; Matrimonio; Relaciones padres-niños.

As perdas são intrínsecas ao processo de constituição e manutenção das famílias ao longo de seu ciclo vital (Silva, 2009), criando desafios adaptativos imediatos e de longo prazo (Walsh \& McGoldrick, 2013). A morte de filhos é considerada especialmente desafiadora, pois inverte a ordem natural desse ciclo (Gonçalves et al., 2014), acarretando maior instabilidade ao sistema familiar. Particularmente, as perdas ocorridas durante a gestação são permeadas por fantasias; além da perda do bebê real, outras perdas se sucedem, como a da gravidez, do bebê imaginado e do exercício da maternidade e paternidade (Koch, Santos, \& Santos, 2012). Apesar de os pais não terem construído um relacionamento concreto com o bebê, o processo de luto após uma perda gestacional não difere em intensidade de outras situações de perda (Kersting \& Wagner, 2012).

Até o momento, não há uma definição consensual na literatura para a terminologia perda gestacional (Delabaere et al., 2014). Uma das denominações usadas é óbito fetal, definido pela Organização Mundial da Saúde (OMS) como morte de um produto da concepção, antes da sua expulsão ou extração completa do corpo da mãe, independentemente da duração da gravidez (Brasil, 2014). Outras expressões no cenário nacional fazem distinções de acordo com o período gestacional em que a perda ocorre, como, por exemplo, abortamento espontâneo, caracterizado pela interrupção da gravidez antes da $20^{\mathrm{a}}$ semana de gestação (Brasil, 2012). Já morte fetal intrauterina é entendida como óbito a partir da $20^{\mathrm{a}}$ semana completa de gestação ou se o feto atingiu peso igual ou superior a 500 gramas e/ou estatura a partir de $25 \mathrm{~cm}$ (Laurenti \& Jorge, 2015). Por fim, o termo natimorto é utilizado para definir fetos que ultrapassaram o estágio de 28 semanas ou mais no útero, mas que não apresentaram quaisquer sinais de vida ao nascer (IBGE, 2010). Assim, a expressão perda gestacional (PG) será utilizada neste estudo para referir-se aos abortamentos espontâneos e mortes fetais ocorridas em qualquer estágio do período gestacional, incluindo o parto (natimortos).

A PG é um fenômeno frequente. Em 2018, a taxa de óbitos fetais no país foi 10,4/1000 nascimentos (Serafim et al., 2021). Além da alta prevalência, a PG é considerada uma perda socialmente negada (Silva, 2009; Walsh \& McGoldrick, 2013; 
Worden, 2013), ou seja, uma experiência para a qual não existe reconhecimento social que a legitime como digna de ser vivenciada. A negação social da PG pode interferir não apenas na dinâmica familiar, mas também no seu tratamento: apenas uma pequena porcentagem daqueles que vivenciam essa situação busca ou recebe tratamento psicológico (Kersting et al., 2011). A invisibilidade das repercussões de uma perda dessa natureza implica, inclusive, na ausência de políticas públicas e ações para a sua redução e a qualificação da sua assistência (Brasil, 2014).

O impacto provocado pela PG é sentido por toda a família. Embora as pesquisas sobre luto abordem o processo numa perspectiva predominantemente individual, é necessário avaliar também o impacto da morte na família enquanto sistema relacional (Walsh \& McGoldrick, 2013). As relações parentais podem ser modificadas, especialmente frente a uma nova gestação (Bowlby, 2004), já que mais de $80 \%$ dos pais que sofreram uma PG terão uma nova gravidez (DeBackere et al., 2008). Igualmente, a vivência dessa perda pode repercutir na relação conjugal (Walsh \& McGoldrick, 2013), considerando, por exemplo, o desafio de lidar com as diferenças de resposta emocional e comportamental de cada cônjuge frente ao luto (Albuquerque et al., 2018; Mcgee et al., 2018).

No Brasil, estudos investigaram a percepção de mulheres em relação à PG (FariaSchützer et al., 2014; Lemos \& Cunha, 2015; Oishi, 2014; Rios et al., 2016; Santos et al., 2004), sendo escassos aqueles que consideraram os impactos dessa vivência na família (Muza et al., 2013). Neste último estudo, foram entrevistadas cinco famílias enlutadas de Brasília, tendo os autores concluído que o apoio social recebido influenciou positivamente a vivência do processo de luto.

O estudo de Paris et al. (2016) avaliou mulheres brasileiras e canadenses que vivenciaram óbito fetal. A prevalência de luto complicado, definido como sofrimento intenso, persistente, angustiante e incapacitante, e como uma dificuldade de rompimento de uma relação significativa ao longo da vida, foi maior entre as brasileiras, em comparação às canadenses. Segundo o estudo, relatar insatisfação conjugal esteve associado a maiores índices de luto complicado. Por sua vez, Morelli e Scorsolini-Comin (2016) entrevistaram cinco casais que haviam perdido um filho por diferentes razões (acidente automobilístico, adoecimento e aborto espontâneo). A morte do filho despertou tanto a rejeição do parceiro em alguns casos/momentos, como maior intimidade. Notou-se maior adaptação nos casais que se ajudaram mutuamente, o que enfatiza a conjugalidade como um recurso importante no enfrentamento da morte.

O aprofundamento das discussões sobre o tema se mostra relevante para profissionais nos contextos da clínica e da assistência em saúde, e para o aperfeiçoamento das intervenções e políticas públicas de saúde mental em torno da parentalidade. Para tanto, é oportuno mapear e sistematizar os resultados de pesquisas científicas nacionais e internacionais e indicar possíveis alternativas para futuras investigações de um fenômeno frequente e impactante a nível individual e relacional. Nesse sentido, o objetivo deste estudo foi analisar a produção científica nacional e internacional no período 2006 a 2021 sobre as repercussões da perda gestacional na conjugalidade e na parentalidade subsequentes à perda. 


\section{MÉTODO}

\section{DELINEAMENTO}

Trata-se de uma revisão sistemática, pesquisa através da qual se pode compilar evidências relacionadas a um tema específico, mediante a aplicação de métodos explícitos e sistematizados de busca, apreciação crítica e síntese da informação selecionada, auxiliando na orientação para futuras investigações (Sampaio \& Mancini, 2007). Para a realização do estudo, foram adotados os seguintes procedimentos: 1) Definição da questão/tema; 2) Estabelecimento dos critérios de busca e seleção dos estudos; 3) Busca e seleção dos estudos; 4) Análise das características metodológicas e dos resultados dos estudos; e 5) Apresentação e discussão dos resultados (Sampaio \& Mancini, 2007). Considerou-se, em todo o processo, as orientações contidas no Preferred Reporting Items for Systematic Reviews and Meta-Analyses - PRISMA (Moher et al., 2009).

\section{PROCEDIMENTOS DE BUSCA}

A pesquisa de artigos foi realizada nas bases de dados Pubmed, PsycNET e Portal BVS (composto pelas bases Scielo, LILACS, Medline, entre outras). Foram considerados selecionáveis apenas estudos empíricos, publicados nos idiomas inglês, espanhol e português, no período de janeiro de 2006 a março de 2021. Foram utilizados os descritores Fetal death OR Miscarriage OR Stillbirth AND (a) Marriage ou (b) Parent-child relations, conforme a plataforma Decs/BVS de Descritores em Saúde. No Portal BVS, as buscas também foram realizadas com os sinônimos em português, conforme segue: Morte fetal OR Aborto espontâneo OR Natimorto AND (a) Casamento ou (b) Relações pais-filho. A expressão perda gestacional não foi utilizada, por não constar no Decs/BVS.

Foram considerados como critérios de exclusão estudos teóricos, duplicação de registros, capítulo, livro, Dissertação de Mestrado e Tese de Doutorado, estudos indisponíveis na íntegra, publicações não avaliadas por pares e fora do período estipulado. Os seguintes filtros de busca foram acionados nas bases de dados, sempre que disponíveis: período 2006 a 2021 e artigos publicados em periódicos revisados por pares. Ainda, foi considerado como critério de exclusão estudos com amostras que haviam vivenciado outros tipos de perda que não PG, exceto se os diferentes tipos de perdas foram analisados separadamente, comparando grupos, por exemplo. Todo o processo de busca e seleção dos artigos pode ser observado no fluxograma apresentado na Figura 1.

\section{ANÁLISE DOS DADOS}

Com base no objetivo desta revisão, duas categorias foram definidas a priori: repercussões da PG na conjugalidade e repercussões da PG na parentalidade. Os resultados dos artigos selecionados foram submetidos à análise temática reflexiva (Braun et al., 2019), um processo sistemático e recursivo de identificação, organização e esclarecimento de padrões de sentido (temas) de um conjunto de dados. Este método é desenvolvido em seis estágios: familiarização com os dados; codificação; busca de temas; revisão dos temas; definição e nomeação de temas; e escrita integrativa (Braun et al., 2019). Este método foi utilizado para a definição dos eixos temáticos dentro de cada uma das duas categorias previamente definidas. 


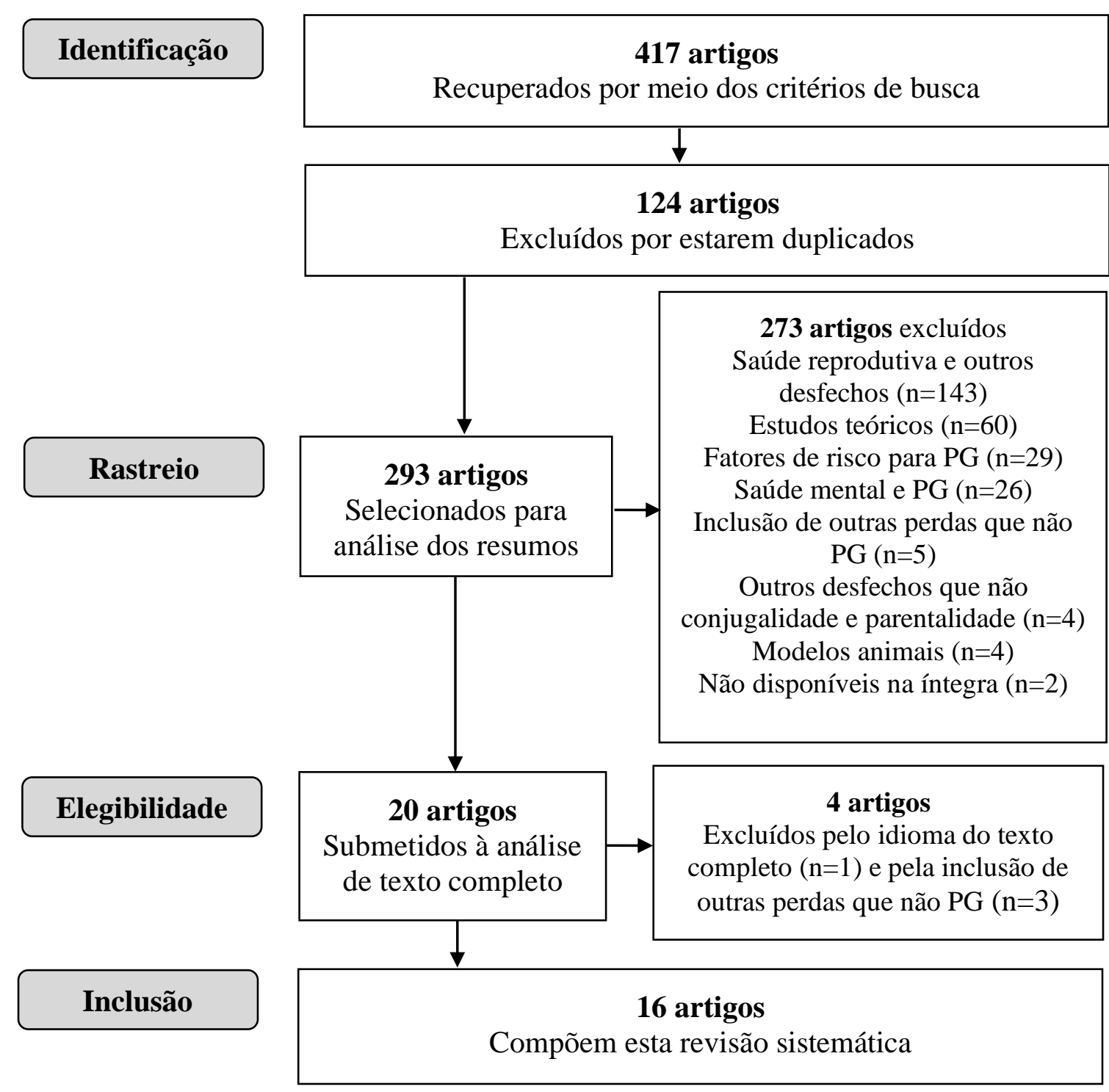

Figura 1

Fluxograma de rastreio, seleção e recuperação do corpus do estudo.

\section{RESUltados}

A partir das buscas, foram recuperados 417 registros, na seguinte distribuição: Pubmed $(n=263)$, Portal BVS $(n=136)$ e PsycNET $(n=18)$. Estes registros foram inseridos no software Rayyan (Ouzzani et al., 2016) para gerenciamento. Após a exclusão de 124 registros repetidos, restaram 293 para análise. Destes, 273 foram excluídos por razões metodológicas e/ou temáticas: (a) Investigação de temas diversos de saúde reprodutiva e outros desfechos associados, sem relação com o foco do presente estudo, como casamento consanguíneo, síndromes genéticas e malformações fetais, comportamentos em saúde durante a gestação, fatores associados à infertilidade, etc.; (b) Estudos teóricos, incluindo revisões sistemáticas, narrativas, meta-análises, cartas/correspondências e opiniões; (c) Análise de causas e/ou fatores de risco para a ocorrência da PG; (d) Estudos que avaliaram exclusivamente o impacto 
da PG na saúde mental materna e/ou paterna, sem considerar aspectos da conjugalidade e parentalidade; (e) Estudos que incluíram outros tipos de perda, tais como aborto induzido, perdas neonatais e morte de filhos maiores, mesmo em conjunto com PG, sem discriminá-las; (f) Estudos que focaram em outros desfechos que não a conjugalidade e parentalidade, como os impactos sociais da perda e na relação pais-avós; (g) Estudos que usaram modelos animais (ratos e gado); e (h) Artigos indisponíveis na íntegra. Com essas exclusões, 20 estudos foram elegíveis para análise. Desses, quatro ainda foram excluídos, seja pelo idioma $(n=1)$, seja por incluir outras perdas na análise $(\mathrm{n}=3)$. Ao final, 16 estudos que compuseram esta revisão.

\section{Tabela 1}

Caracterização dos estudos elegíveis

\begin{tabular}{|c|c|c|c|c|c|}
\hline Autores & Ano & Delineamento & Amostra & Desfecho & País \\
\hline $\begin{array}{l}\text { Kiełek-Rataj } \\
\text { et al. }\end{array}$ & 2020 & $\begin{array}{l}\text { Correlacional } \\
\text { comparativo }\end{array}$ & $\begin{array}{l}90 \text { casais com histórico de } \\
\text { infertilidade ou PG }\end{array}$ & $\begin{array}{l}\text { Abertura, comunicação e } \\
\text { satisfação conjugal }\end{array}$ & Polônia \\
\hline $\begin{array}{l}\text { Horstman } \\
\text { et al. }\end{array}$ & 2019 & $\begin{array}{l}\text { Descritivo } \\
\text { qualitativo }\end{array}$ & $\begin{array}{l}45 \text { homens casados que } \\
\text { viveram uma } \mathrm{PG}\end{array}$ & $\begin{array}{l}\text { Comunicação sobre a PG } \\
\text { por meio de metáforas }\end{array}$ & EUA \\
\hline $\begin{array}{l}\text { Horstman e } \\
\text { Holman }\end{array}$ & 2018 & Correlacional & $\begin{array}{c}183 \text { casais que viveram } \\
\text { uma PG }\end{array}$ & $\begin{array}{c}\text { Comunicação e } \\
\text { satisfação conjugal, e } \\
\text { percepção de } \\
\text { parentalidade do bebê } \\
\text { perdido }\end{array}$ & EUA \\
\hline $\begin{array}{l}\text { Üstündağ - } \\
\text { Budak et al. }\end{array}$ & 2015 & $\begin{array}{l}\text { Descritivo } \\
\text { qualitativo }\end{array}$ & $\begin{array}{c}6 \text { mulheres com histórico } \\
\text { de PG na primeira } \\
\text { gestação, que tiveram filho } \\
\text { após }\end{array}$ & $\begin{array}{c}\text { Experiência da } \\
\text { parentalidade após PG }\end{array}$ & Inglaterra \\
\hline Kinsey et al. & 2014 & $\begin{array}{l}\text { Longitudinal } \\
\text { correlacional }\end{array}$ & $\begin{array}{c}2798 \text { mães com histórico } \\
\text { de PG acessadas a } 1,6 \text { e } 12 \\
\text { meses de vida do novo } \\
\text { bebê }\end{array}$ & Vinculação mãe-bebê & EUA \\
\hline $\begin{array}{l}\text { Campbell- } \\
\text { Jackson et al. }\end{array}$ & 2014 & $\begin{array}{l}\text { Descritivo } \\
\text { qualitativo }\end{array}$ & $\begin{array}{l}7 \text { casais com histórico de } \\
\text { PG que tiveram o primeiro } \\
\text { filho vivo }\end{array}$ & $\begin{array}{c}\text { Experiência da } \\
\text { parentalidade após PG }\end{array}$ & Inglaterra \\
\hline $\begin{array}{l}\text { Mehran } \\
\text { et. al. }\end{array}$ & 2013 & $\begin{array}{l}\text { Correlacional } \\
\text { comparativo }\end{array}$ & $\begin{array}{l}100 \text { gestantes com e sem } \\
\text { histórico de PG }\end{array}$ & Apego materno-fetal & Irã \\
\hline $\begin{array}{l}\text { Sugiura- } \\
\text { Ogasawara } \\
\text { et. al. }\end{array}$ & 2013 & $\begin{array}{l}\text { Correlacional } \\
\text { comparativo }\end{array}$ & $\begin{array}{l}2733 \text { mulheres com e sem } \\
\text { histórico de PGR }\end{array}$ & Status de relacionamento & Japão \\
\hline $\begin{array}{l}\text { Kagami } \\
\text { et. al. }\end{array}$ & 2012 & Correlacional & $\begin{array}{c}76 \text { casais que vivenciaram } \\
\text { PGR }\end{array}$ & $\begin{array}{l}\text { Qualidade conjugal e } \\
\text { estresse associado à PGR }\end{array}$ & Japão \\
\hline $\begin{array}{l}\text { Erlandsson } \\
\text { et al. }\end{array}$ & 2010 & $\begin{array}{l}\text { Descritivo } \\
\text { qualitativo }\end{array}$ & $\begin{array}{c}16 \text { pais e mães um ano } \\
\text { após PG }\end{array}$ & $\begin{array}{c}\text { Percepção parental sobre } \\
\text { suporte dado aos outros } \\
\text { filhos }\end{array}$ & Suíça \\
\hline Gold et al. & 2010 & $\begin{array}{l}\text { Longitudinal } \\
\text { comparativo }\end{array}$ & $\begin{array}{l}3707 \text { mulheres que tiveram } \\
7770 \text { gestações com e sem } \\
\text { PG }\end{array}$ & Status de relacionamento & EUA \\
\hline Turton et al. & 2009 & $\begin{array}{l}\text { Longitudinal } \\
\text { comparativo }\end{array}$ & $\begin{array}{l}52 \text { mães com e } 51 \text { sem } \\
\text { histórico de PG e seus } \\
\text { filhos de } 6-8 \text { anos }\end{array}$ & $\begin{array}{l}\text { Vulnerabilidade } \\
\text { psicológica da criança } \\
\text { nascida após PG }\end{array}$ & Inglaterra \\
\hline $\begin{array}{l}\text { De Pascalis } \\
\quad \text { et al. }\end{array}$ & 2008 & $\begin{array}{l}\text { Correlacional } \\
\text { comparativo }\end{array}$ & $\begin{array}{l}53 \text { casais que tiveram } \\
\text { gestação gemelar e } \\
\text { perderam apenas um dos } \\
\text { bebês e } 106 \text { casais com }\end{array}$ & $\begin{array}{l}\text { Vulnerabilidade } \\
\text { psicológica da criança } \\
\text { nascida após PG }\end{array}$ & Itália \\
\hline
\end{tabular}




\begin{tabular}{|c|c|c|c|c|c|}
\hline Autores & Ano & Delineamento & Amostra & Desfecho & País \\
\hline \multicolumn{6}{|c|}{ gestação única sem PG } \\
\hline Price & 2008 & $\begin{array}{c}\text { Correlacional } \\
\text { comparativo }\end{array}$ & $\begin{array}{c}10.688 \text { mães de crianças } \\
\text { com um ano de vida, com e } \\
\text { sem histórico de PG }\end{array}$ & Interação mãe-criança & EUA \\
\hline Hughes et al. & 2006 & $\begin{array}{l}\text { Longitudinal } \\
\text { comparativo }\end{array}$ & $\begin{array}{l}31 \text { mães com histórico de } \\
\text { PG acessadas na gestação } \\
\text { subsequente e aos } 12 \\
\text { meses do novo bebê }\end{array}$ & $\begin{array}{l}\text { Apego adulto e apego } \\
\text { com o filho subsequente }\end{array}$ & Inglaterra \\
\hline $\begin{array}{l}\text { Tsartsara e } \\
\text { Johnson }\end{array}$ & 2006 & $\begin{array}{l}\text { Longitudinal } \\
\text { comparativo }\end{array}$ & $\begin{array}{l}35 \text { mulheres com e sem } \\
\text { histórico de PG acessadas } \\
\text { no primeiro e terceiro } \\
\text { trimestre da gestação } \\
\text { subsequente }\end{array}$ & $\begin{array}{l}\text { Ansiedade relacionada à } \\
\text { gestação e apego } \\
\text { materno-fetal }\end{array}$ & Inglaterra \\
\hline
\end{tabular}

Nota. PG= Perda gestacional; PGR= Perda gestacional recorrente (duas ou mais consecutivas).

Nenhum estudo brasileiro foi encontrado. Quatro artigos apresentaram metodologia qualitativa descritiva e doze estudos quantitativos adotaram delineamento correlacional e/ou comparativo, sendo cinco destes longitudinais. Quanto ao país de origem, apesar da diversidade, predominaram estudos norte-americanos $(n=5)$ e ingleses $(n=5)$. Seis estudos consideraram casais como participantes; apenas um incluiu somente os homens, pois os demais avaliaram somente as mulheres.

A análise temática dos resultados dos estudos selecionados originou três eixos temáticos para cada uma das duas categorias, conforme apresentado na Tabela 2.

\section{Tabela 2}

\section{Categorias, eixos temáticos e resultados dos estudos}

\begin{tabular}{|c|c|c|c|}
\hline Categoria & Eixos temáticos & Resultados & Estudos \\
\hline \multirow{5}{*}{$\begin{array}{c}\text { Repercussões } \\
\text { na } \\
\text { conjugalidade }\end{array}$} & \multirow{2}{*}{$\begin{array}{l}\text { Qualidade/ } \\
\text { satisfação } \\
\text { conjugal }\end{array}$} & $\begin{array}{l}\text { Qualidade conjugal associou-se negativamente } \\
\text { ao estresse conjugal relacionado à PGR em } \\
\text { homens e mulheres. Baixa qualidade conjugal } \\
\text { associou-se a pior ajuste psicológico nas } \\
\text { mulheres, mas não nos homens. }\end{array}$ & (Kagami et al., 2012) \\
\hline & & $\begin{array}{l}\text { Satisfação conjugal foi mais baixa nas mulheres } \\
\text { inférteis comparadas com as mulheres que } \\
\text { tiveram uma PG. Percepção de abertura no } \\
\text { parceiro está relacionada com satisfação } \\
\text { conjugal nas mulheres. }\end{array}$ & $\begin{array}{c}\text { (Kiełek-Rataj et al., } \\
\text { 2020) }\end{array}$ \\
\hline & $\begin{array}{l}\text { Término do } \\
\text { relacionamento }\end{array}$ & $\begin{array}{l}\text { Mulheres com história de PG ou PGR } \\
\text { apresentaram risco maior de } \\
\text { separação/divórcio, em comparação com } \\
\text { aquelas que tiveram uma gestação bem- } \\
\text { sucedida. }\end{array}$ & $\begin{array}{c}\text { (Gold et al., 2010; } \\
\text { Sugiura-Ogasawara et } \\
\text { al., 2013) }\end{array}$ \\
\hline & \multirow[t]{2}{*}{$\begin{array}{l}\text { Comunicação } \\
\text { conjugal }\end{array}$} & $\begin{array}{l}\text { Comunicação baseada na validação da } \\
\text { perspectiva do outro associou-se com bem-estar } \\
\text { individual e conjugal, principalmente em } \\
\text { homens. Identificação enquanto pais da criança } \\
\text { perdida moderou os efeitos da comunicação no } \\
\text { bem-estar conjugal. }\end{array}$ & $\begin{array}{c}\text { (Horstman \& Holman, } \\
\text { 2018) }\end{array}$ \\
\hline & & $\begin{array}{c}\text { Sobre seu papel na } \mathrm{PG} \text {, os homens utilizaram as } \\
\text { metáforas rocha, guardião, reparador e } \\
\text { personagem secundário. }\end{array}$ & $\begin{array}{l}\text { (Horstman et al., } \\
\text { 2019) }\end{array}$ \\
\hline
\end{tabular}




\begin{tabular}{|c|c|c|c|}
\hline Categoria & Eixos temáticos & Resultados & Estudos \\
\hline \multirow{8}{*}{$\begin{array}{l}\text { Repercussões } \\
\text { na } \\
\text { parentalidade }\end{array}$} & $\begin{array}{l}\text { Gestação } \\
\text { subsequente }\end{array}$ & $\begin{array}{c}\text { Não houve diferença na vinculação materno- } \\
\text { fetal entre gestantes com e sem histórico de PG. } \\
\text { Gestantes com PG pontuaram menos em } \\
\text { diferenciação de si com o feto e apresentaram } \\
\text { mais ansiedade relacionada à gestação. No } \\
\text { terceiro trimestre, a ansiedade reduziu de } \\
\text { maneira significativa. }\end{array}$ & $\begin{array}{l}\text { (Mehran et al., 2013; } \\
\text { Tsartsara \& Johnson, } \\
\text { 2006) }\end{array}$ \\
\hline & \multirow{5}{*}{$\begin{array}{l}\text { Relação com filho } \\
\text { subsequente }\end{array}$} & $\begin{array}{c}\text { Não houve diferença entre histórico de PG ou } \\
\text { PGR e dificuldades de vinculação e interação } \\
\text { materna com o filho subsequente e nem na } \\
\text { percepção parental sobre a relação com o novo } \\
\text { bebê. }\end{array}$ & $\begin{array}{l}\text { (Kinsey et al., 2014; } \\
\text { Price, 2008; } \\
\text { Campbell-Jackson et } \\
\text { al., 2014) }\end{array}$ \\
\hline & & $\begin{array}{l}\text { Mães com histórico de PG reportaram mais } \\
\text { dificuldades em seus filhos, apresentaram } \\
\text { interação de menor qualidade e mais chances de } \\
\text { apresentar sintomas de depressão pós-parto. }\end{array}$ & $\begin{array}{l}\text { (Turton et al., 2009; } \\
\text { Kinsey et al., 2014) }\end{array}$ \\
\hline & & $\begin{array}{l}\text { Mães com resposta desorganizada à perda, mas } \\
\text { que não tinham apego desorganizado com o } \\
\text { filho subsequente, apresentaram mais depressão } \\
\text { e pensamentos intrusivos na gestação e } 1 \text { ano } \\
\text { após. }\end{array}$ & (Hughes et al., 2006) \\
\hline & & $\begin{array}{l}\text { Famílias com histórico de perda de um dos } \\
\text { gêmeos perceberam os filhos sobreviventes } \\
\text { como menos vulneráveis em relação ao grupo } \\
\text { controle sem PG. Mulheres com PGR } \\
\text { envolveram-se mais nas atividades de contar } \\
\text { histórias e cantar para o filho subsequente. }\end{array}$ & $\begin{array}{l}\text { (De Pascalis et al., } \\
\text { 2008; Price, 2008) }\end{array}$ \\
\hline & & $\begin{array}{l}\text { Pais relataram ansiedade, expectativas irreais e } \\
\text { culpa, mas também crescimento após a perda, } \\
\text { que, respectivamente, influenciaram negativa e } \\
\text { positivamente a relação com novo bebê. }\end{array}$ & $\begin{array}{l}\text { (Campbell-Jackson et } \\
\text { al., 2014; Üstündağ - } \\
\text { Budak et al., 2015) }\end{array}$ \\
\hline & \multirow{2}{*}{$\begin{array}{l}\text { Processo de luto } \\
\text { familiar }\end{array}$} & $\begin{array}{c}\text { A ausência de filhos foi relacionada à maior } \\
\text { ansiedade nas mulheres, enquanto histórico de } \\
\text { PGR associou-se a mais sintomas depressivos } \\
\text { nas mulheres. }\end{array}$ & $\begin{array}{c}\text { (Kagami et al., 2012; } \\
\text { Price, 2008) }\end{array}$ \\
\hline & & $\begin{array}{l}\text { A participação dos irmãos no processo de luto } \\
\text { por PG pareceu positiva para pais e filhos, pelo } \\
\text { compartilhar da experiência de luto e da } \\
\text { memória da criança perdida. }\end{array}$ & $\begin{array}{c}\text { (Erlandsson et al., } \\
\text { 2010) }\end{array}$ \\
\hline
\end{tabular}

Nota. $\mathrm{PG}=$ Perda gestacional $\mathrm{PGR}=$ Perda gestacional recorrente (duas ou mais consecutivas)

Os resultados apontaram que a maior parte dos estudos $(n=11)$ avaliou as relações entre histórico de PG e parentalidade, enquanto seis avaliaram a conjugalidade após este evento. Apenas um estudo (Kagami et al., 2012) apresentou tanto as relações entre PG e parentalidade quanto entre $\mathrm{PG}$ e conjugalidade.

Em relação à conjugalidade, mesmo considerando diferentes delineamentos e amostras, foi possível notar que a PG parece se configurar como fator de risco para a qualidade/satisfação conjugal, podendo levar ao término do relacionamento. Por outro lado, aspectos da comunicação conjugal e a identificação enquanto pais da criança perdida parecem estar relacionados a melhores índices de bem-estar no casal. Os estudos ainda indicam uma 
tendência de que PGR e infertilidade possam ter impactos ainda mais negativos na conjugalidade e mesmo no processo de luto, embora novos estudos sejam necessários para confirmar esta hipótese.

Quanto à parentalidade, não se observou diferença na vinculação materno-fetal durante a gestação subsequente, avaliada em apenas dois estudos, porém, estes também apontaram para um possível aumento nos sintomas depressivos e ansiosos no período gestacional subsequente à PG. No tocante à relação com o filho subsequente, foram encontrados resultados contrastantes: tanto houve ausência de associação entre histórico de PG e dificuldade de vinculação e interação materna com o novo bebê, quanto presença de dificuldades com os filhos e interação de menor qualidade. Ainda, alguns estudos apontaram a associação entre vivência de PG prévia e aspectos positivos na relação com o filho subsequente, como percepção de menor vulnerabilidade da criança e mais engajamento em atividades com ela. Por fim, de outra perspectiva, a presença de outros filhos pareceu ser protetora aos pais enlutados por PG.

\section{DISCUSSÃo}

Esta revisão buscou analisar a produção científica sobre as repercussões da PG na conjugalidade e na parentalidade subsequentes à perda. Foram encontradas tanto repercussões negativas quanto positivas e mesmo ausência de relação entre estes aspectos. Resultados contrastantes foram encontrados principalmente em relação à parentalidade, algo já apontado na literatura (Lee et al., 2017). Tais achados sugerem que, apesar de a PG ser um evento com grande potencial estressor, diferentes fatores interferem no exercício da parentalidade e na conjugalidade subsequentes, favorecendo ou dificultando a vivência do processo de luto decorrente da PG e modificando as suas possíveis repercussões. Os resultados encontrados por Price (2008), Kinsey et al. (2014), Campbell-Jackson et al. (2014), De Pascalis et al. (2008), Horstman e Holman (2018) e Üstündağ-Budak et al. (2015) parecem corroborar esta reflexão.

Entretanto, importante destacar que a maior parte dos estudos incluídos nesta revisão buscou investigar os riscos relacionados à conjugalidade e à parentalidade após a PG. Neste sentido, é preciso distinguir entre problemas psicossociais e processos de enfrentamento de eventos de vida estressores (Lee \& Rowlands, 2015). No contexto da PG, as autoras indicam que os esforços para lidar com emoções difíceis e construir significado para a perda devem ser vistos como trajetórias de resiliência, que envolvem tarefas emocionalmente desafiadoras, e não necessariamente problemas conjugais ou parentais.

A adaptação ao luto é um processo que ocorre através do tempo, moldado por fatores de risco e proteção ambientais (em nível familiar, comunitário e cultural) e individuais (Sandler et al., 2007). Dessa forma, é possível pensar a PG como uma experiência que poderá promover tanto riscos quanto crescimento, dependendo de complexos fatores que se interrelacionam sistemicamente. Assim, as pesquisas devem também ter como foco fatores de proteção e processos de crescimento pós-traumático.

Entre os estudos analisados, a ausência de filhos e a vivência de múltiplas perdas apresentaram-se como aspectos que tendem a gerar repercussões negativas na conjugalidade e na vivência do luto familiar (Kiełek-Rataj et al., 2020; Kagami et al., 2012; Price, 2008; Sugiura-Ogasawara et al., 2013). Outros estudos já apontaram repercussões negativas de perdas recorrentes, infertilidade e ausência de filhos na saúde mental de mulheres (He et al., 2019), porém, as repercussões na conjugalidade merecem mais investigações. Por outro lado, o fato de engravidar e ter filhos após uma PG não é por si só promotor de 
resiliência, uma vez que a gestação subsequente pode ser permeada por sintomas de depressão e ansiedade (DeBackere et al., 2008). Ainda, mulheres que tiveram filhos após uma perda com intenções claras de substituir a criança perdida apresentaram reverberações negativas desse acontecimento na sua saúde mental (O’Leary, 2004; Vidal, 2010), o que, por sua vez, pode levar a maiores dificuldades parentais e conjugais.

Um dos estudos aqui revisados (Horstman \& Holman, 2018) apontou que a identificação enquanto pais da criança perdida moderou os efeitos da comunicação no bemestar conjugal. Isso leva a crer que a visão do filho perdido como único e de si como pais possivelmente auxilia na vivência do luto, impedindo maiores dificuldades na relação com o filho subsequente à perda. Neste sentido, segundo Markin (2018), o conceito de mentalização auxilia na compreensão das razões pelas quais alguns pais conseguem lidar bem com o desafio de vincular-se a um bebê enquanto vivem o luto por outro. Para esta autora, a capacidade de mentalizar (a) afetos associados ao trauma e à perda, (b) as duas relações de apego como distintas, (c) estados mentais múltiplos e conflitantes e (d) experiências de apego precoce relacionadas à perda e ao trauma, podem ajudar os pais a viver ambas as experiências, evitando problemas de apego com o filho subsequente.

Quanto às repercussões da PG no nível conjugal, os resultados sugerem que esse evento possa ser um fator de risco para a conjugalidade. O maior risco de dissolução da relação e os baixos índices de qualidade/satisfação conjugal entre casais com história de PG podem ser explicados, ao menos parcialmente, pelas diferentes respostas de homens e mulheres à PG. A discrepância na vivência do processo de luto entre os parceiros apresenta-se como fator de potencial dificuldade para a interação conjugal (Doka \& Martin, 2011), enquanto o enfrentamento diádico apresenta-se como fator de proteção para a vivência do luto (Albuquerque et al., 2018; Mcgee et al., 2018).

Embora a experiência da PG seja diferente entre os gêneros (Doka \& Martin, 2011), a literatura tem focalizado mais frequentemente as reações e enfrentamento das mulheres, em detrimento dos homens. Na presente revisão, mais da metade $(n=9)$ dos estudos analisados avaliaram apenas mulheres. Apenas seis estudos consideraram o casal como participantes, sendo que, destes, apenas três avaliaram as diferenças entre as respostas dos cônjuges à PG (Horstman \& Holman, 2018; Kagami et al., 2012; Kiełek-Rataj et al., 2020).

A comunicação conjugal é um dentre muitos fatores em que as diferenças de gênero se manifestam no enfrentamento da perda. Conforme o estudo de Kiełek-Rataj e colegas (2020) a percepção de abertura no parceiro relacionou-se com satisfação conjugal nas mulheres. Já de acordo com Horstman e Holman (2018), uma comunicação baseada na validação da perspectiva do outro associou-se com bem-estar individual e conjugal, principalmente para os homens. Assim, homens que perceberam a confirmação da sua perspectiva sobre a perda pelas esposas avaliaram a experiência e o casamento de forma mais positiva. Esses resultados sugerem a importância da validação social na vivência do luto (Bellet et al., 2018) que ocorre principalmente por meio da comunicação.

Ainda, os homens utilizaram as metáforas "rocha, guardião, reparador e personagem secundário" para referirem-se ao seu papel na vivência da PG (Horstman et al., 2019). Outros estudos já apontaram que os parceiros se veem no papel de fornecer suporte para as mulheres nas situações de perda (Due et al., 2017), embora possuam necessidades diferentes das delas (Ellis et al., 2016). Este papel secundário em que os homens se colocam e são colocados implica na eventual falta de reconhecimento de seu processo de luto, gerando impactos em sua saúde mental e comportamento (Bellet et al., 2018; Due et al., 2017). Neste sentido, a falta de reconhecimento social afeta mulheres e homens que vivem uma PG e, consequentemente, a sua relação conjugal. 
Considerando tais diferenças e as especificidades da PG, intervenções em saúde que contemplem ambos os cônjuges (como a terapia de casal, por exemplo) parecem ser adequadas para lidar com o estresse psicológico e psicossocial relacionado a este acontecimento, visando fortalecer o relacionamento conjugal, para que os parceiros se auxiliem no processo de adaptação à perda (Kagami et al., 2012 O’Leary, 2004). Nenhum estudo de descrição ou avaliação de intervenções neste âmbito foi encontrado, sugerindo uma potencial lacuna na literatura.

Percebe-se que as investigações científicas sobre as relações conjugais e parentais após uma PG têm investigado principalmente fatores de risco (como término do relacionamento e dificuldades de relacionamento com o bebê subsequente). Contudo, a PG precisa ser encarada como um evento biopsicossocial, cultural, heterogêneo e situacional (Sijpt, 2010), abordado não somente do ponto de vista do risco epidemiológico, mas também do ponto de vista da experiência pessoal (Price, 2008), identificando-se processos de risco e proteção. Tais características são compatíveis com metodologias qualitativas, que foram minoritariamente empregadas nos estudos incluídos nesta revisão.

Os resultados contrastantes aqui encontrados sugerem a necessidade de compreender também os processos de crescimento pós-traumático que podem ser acionados após a PG, que podem envolver a comunicação conjugal. Ainda, alguns aspectos têm sido negligenciados nas pesquisas, entre eles, as repercussões da perda na perspectiva dos homens (pais) e a avaliação de intervenções psicológicas neste contexto. $\mathrm{O}$ desafio das futuras pesquisas e intervenções reside na abordagem e compreensão da complexa interação entre variáveis pessoais, familiares e socioculturais que geram diferentes respostas ao evento da perda (Price, 2008).

Quanto às limitações do presente estudo, é possível considerar a diversidade de termos existentes para tratar do assunto, que pode ter dificultado o acesso a estudos potencialmente elegíveis. Neste sentido, coloca-se como uma necessidade a padronização dos termos no cenário nacional, a fim de que se possa dirimir tal obstáculo metodológico em futuras revisões sistemáticas. Sugere-se a inclusão de termos mais adequados à realidade das pesquisas em Ciências Humanas aplicadas à saúde no DeCS (como perda gestacional, por exemplo), para que seja possível proceder a buscas mais acuradas nas bases de dados pesquisadas. Por fim, ressalta-se que os aspectos conjugais e parentais foram abordados de forma separada em algumas seções desse artigo, por um fim meramente didático, uma vez que estes processos se inter-relacionam e se influenciam mutuamente.

\section{CONSIDERAÇÕES FINAIS}

Por meio da análise dos estudos incluídos na presente revisão sistemática, verificou-se que a maior parte deles buscou investigar as repercussões da PG na parentalidade. Não foram encontrados relatos de dificuldade de vinculação durante a gestação. Após o nascimento dos filhos subsequentes, os resultados apontaram tanto presença quanto ausência de dificuldades na relação pais-criança. Esses achados contrastantes demonstram a necessidade de investigação dos fatores de proteção e dos processos de crescimento pós-traumático acionados após a PG, para além dos fatores de risco. Ainda, a presença de filhos pareceu ser um fator protetor à vivência do luto para os pais, enquanto sua ausência pareceu sobrecarregar a relação conjugal. A PG apresentou-se como fator de risco à conjugalidade (qualidade/satisfação conjugal e término do relacionamento). Contudo, a comunicação conjugal emergiu como aspecto protetivo, merecendo mais enfoque na produção científica. 
Identificou-se também a necessidade de pesquisas sobre as repercussões da PG nos homens, incluindo metodologias qualitativas ou mistas, bem como a avaliação de intervenções.

Nessa perspectiva, os resultados do presente estudo indicam que a terapia de casal e de família pode ser um espaço profícuo para a realização de intervenções com pais enlutados diante de uma PG, para que possam lidar conjuntamente com o estresse relacionado a esse evento, prevenindo possíveis dificuldades conjugais e parentais decorrentes da perda. Sugerese, então, como possível campo de investigação, os processos de resiliência familiar envolvidos na adaptação à $\mathrm{PG}$, de maneira a adotar um enfoque positivo na abordagem do luto. A compreensão do fenômeno sob tal perspectiva poderá qualificar a atuação profissional diante dessa situação. Por fim, destaca-se a ausência de estudos brasileiros sobre o tema, o que indica a necessidade de evidências empíricas que subsidiem a teoria e a prática profissional, com base nas especificidades da cultura brasileira.

\section{REFERÊNCIAS}

Albuquerque, S., Narciso, I., \& Pereira, M. (2018). Dyadic coping mediates the relationship between parents' grief and dyadic adjustment following the loss of a child. Anxiety, Stress, \& Coping, 31(1), 93-106. http://doi.org/10.1080/10615806.2017.1363390

Bellet, B. W., Holland, J. M. \& Neimeyer, R. A. (2018): The Social Meaning in Life Events Scale (SMILES): A preliminary psychometric evaluation in a bereaved sample. Death Studies, 43(2), 103-112. http://doi.org/10.1080/07481187.2018.1456008

Bowlby, J. (2004). Perda: Tristeza e depressão. Martins Fontes. (Original publicado em 1980) Brasil. (2012). Gestação de alto risco: Manual Técnico. Ministério da Saúde. Brasília: Autor. Disponível em: www.saude.gov.br/saudemulher

Brasil. (2014). Atenção à saúde do recém-nascido: Guia para profissionais de saúde Cuidados Gerais. Ministério da Saúde. Brasília: Autor. Disponível em: http://bvsms.saude.gov.br/bvs/publicacoes/atencao_saude_recem_nascido_v1.p df

Braun, V., Clark, V., Hayfield, N., \& Terry, G. (2019). Thematic Analysis. In Liamputtong, P. (Ed.), Handbook of research methods in health social sciences (pp. 843-860). Springer. https://doi.org/10.1080/2159676X.2019.1628806

Campbell-Jackson, L., Bezance, J., \& Horsch, A. (2014). "A renewed sense of purpose": Mothers' and fathers' experience of having a child following a recent stillbirth. BMC Pregnancy and Childbirth, 14(423). http://doi.org/10.1186/s12884-014-0423$\mathrm{X}$

Debackere, K. J., Hill, P. D., \& Kavanaugh, K. L. (2008). The parental experience of pregnancy after perinatal loss. JOGNN - Journal of Obstetric, Gynecologic, and Neonatal Nursing, 37(5), 525-537. http://doi.org/10.1111/j.1552-6909.2008.00275.x

Delabaere, A., Huchon, C., Lavoue, V., Lejeune, V., Iraola, E., Nedellec, S., ... Deffieux, X. (2014). Definition of pregnancy losses: Standardization of terminology from the French National College of Obstetricians and Gynecologists (CNGOF). Journal de Gynécologie Obstétrique et Biologie de la Reproduction, 43(10), 756-763. http://doi.org/10.1016/j.jgyn.2014.09.010

De Pascalis, L., Monti, F., Agostini, F., Fagandini, P., La Sala, G. B., \& Blickstein, I. (2008). Psychological Vulnerability of Singleton Children After the 'Vanishing' of a Co-Twin Following Assisted Reproduction. Twin Research and Human Genetics, 11(1), 93-98. https://doi.org/10.1375/twin.11.1.93

Doka, K. J., \& Martin, T. L. (2011). Grieving beyond gender: Understanding the ways men 
and women mourn. Routledge. https://doi.org/10.4324/9780203886069

Due, C., Chiarolli, S., \& Riggs, D. W. (2017). The impact of pregnancy loss on men's health and wellbeing: A systematic review. BMC Pregnancy Childbirth, 17(1), 380. http://doi.org/10.1186/s12884-017-1560-9

Ellis, A., Chebsey, C., Storey, C., Bradley, S., Jackson, S., Flenady, V., ... \& Siassakos, D. (2016). Systematic review to understand and improve care after stillbirth: A review of parents' and healthcare professionals' experiences. BMC Pregnancy and Childbirth, 16(1), 1-19. http://doi.org/10.1186/s12884-016-0806-2

Faria-Schützer, D. B., Neto, G. L., Duarte, C. A. M., Vieira, C. M., \& Turato, E. R. (2014). Fica um grande vazio: Relatos de mulheres que experienciaram morte fetal durante a gestação. Estudos Interdisciplinares em Psicologia, 5(2), 113. http://dx.doi.org/10.5433/2236-6407.2014v5n2p113

Gold, K. J., Sen, A., \& Hayward, R. A. (2010). Marriage and cohabitation outcomes after pregnancy loss. Pediatrics, 125(5), e1202-e1207. http://doi.org/10.1542/peds.2009-3081

Gonçalves, J. T. T. (2014). Luto parental em situações de morte inesperada: Reações à perda, estratégias de coping e perceção de qualidade de vida [Dissertação de Mestrado]. Universidade de

Lisboa. https://repositorio.ul.pt/bitstream/10451/18271/1/ulfpie047275_tm_tese.pdf

$\mathrm{He}$, L., Wang, T., Xu, H. et al. (2019). Prevalence of depression and anxiety in women with recurrent pregnancy loss and the associated risk factors. Archives of Gynecology and Obstetrics. 300, 1061-1066. https://doi.org/10.1007/s00404-019-05264-z

Hortsman, H. K. \& Holman, A. (2018). Communicated sense-making after miscarriage: A dyadic analysis of spousal communicated perspective-taking, well-being, and parenting role salience. Health communication, 33(10), 1317-1326. https://doi.org/10.1080/10410236.2017.1351852

Hortsman, H. K., Holman, A., \& McBride, M. C. (2019). Men's use of metaphors to make sense of their spouse's miscarriage: Expanding the communicated sensemaking model. Health Communication, 35(5). https://doi.org/10.1080/10410236.2019.1570430

IBGE-Instituto Brasileiro de Geografia e Estatística. (2010). Registro Civil 2010: Óbito Fetal. Brasilia: Autor. Disponível em: http://www.ibge.gov.br/estatistica/ 2010

Kagami, M., Maruyama, T., Koizumi, T., Miyazaki, K., Nishikawa-Uchida, S., Oda, H., ... Yoshimura, Y. (2012). Psychological adjustment and psychosocial stress among Japanese couples with a history of recurrent pregnancy loss. Human Reproduction, 27(3), 787-794. http://doi.org/10.1093/humrep/der441

Kersting, A., \& Wagner, B. (2012). Complicated grief after perinatal loss. Dialogues in Clinical Neuroscience, 14(2), 187-194. http://doi.org/10.31887/DCNS.2012.14.2/akersting

Kersting, A., Kroker, K., Schlicht, S., \& Wagner, B. (2011). Internet-based treatment after pregnancy loss: Concept and case study. Journal of Psychosomatic Obstetrics \& Gynecology, 32(2), 72-78. http://doi.org/10.3109/0167482X.2011.553974

Kiełek-Rataj, E., Wendołowska, A., Kalus, A., \& Czyzowska, D. (2020). Openness and communication effects on relationship satisfaction in women experiencing infertility or miscarriage: A dyadic approach. International Journal of Environmental Research and Public Health, 17(16), 5721. https://doi.org/10.3390/ijerph17165721

Kinsey, C. B., Baptiste-Roberts, K., Zhu, J., \& Kjerulff, K. H. (2014). Effect of miscarriage history on maternal-infant bonding during the first year postpartum in the First Baby Study: A longitudinal cohort study. BMC Women's Health, 14(1), 83. 
http://doi.org/10.1186/1472-6874-14-83

Koch, C., Santos, C., \& Santos, M. R. (2012). Tradução portuguesa, adaptação e validação da Perinatal Bereavement Grief Scale (PBGS) em mulheres com perda de gravidez. Revista Enfermagem Referência, III Série (6), 123-130. Disponível em: http://www.scielo.mec.pt/pdf/ref/vserIIIn6/serIIIn6a12.pdf

Laurenti, R., \& Jorge, M. H. P. M. (2015). O atestado de óbito: Aspectos médicos, estatísticos, éticos e jurídicos. São Paulo: Conselho Regional de Medicina do Estado de São Paulo (Cremesp). Disponível em: http://www.cremesp.org.br/pdfs/atestado_de_obito.pdf

Lee, C., Rowlands, I. J. (2015). When mixed methods produce mixed results: Integrating disparate findings about miscarriage and women's wellbeing. British Journal of Health Psychology, 20(1), 36-44. http://doi.org/10.1111/bjhp.12121

Lee, L., McKenzie-McHarg, K., Horsch, A. (2017). The impact of miscarriage and stillbirth on maternal-fetal relationships: An integrative review. Journal of Reproductive and Infant Psychology, 35(1), 32-52. http://doi.org/10.1080/02646838.2016.1239249.

Lemos, L. F. S., \& Cunha, A. C. B. (2015). Concepções sobre morte e luto: Experiência feminina sobre a perda gestacional. Psicologia: Ciência e Profissão, 35(4), 1120-1138. https://doi.org/10.1590/1982-3703001582014

Markin, R. D. (2018). "Ghosts" in the womb: A mentalizing approach to understanding and treating prenatal attachment disturbances during pregnancies after loss. Psychotherapy, 55(3), 275-288. https://doi.org/10.1037/pst0000186

Mcgee, K., PettyJohn, M. E., \& Gallus, K. L. (2018). Ambiguous loss: A phenomenological exploration of women seeking support following miscarriage. Journal of Loss and Trauma, 23(6), 516-530. https://doi.org/10.1080/15325024.2018.1484625

Mehran, P., Simbar, M., Shams, J., Ramezani-Tehrani, F., \& Nasiri, N. (2013). History of perinatal loss and maternal-fetal attachment behaviors. Women and Birth, 26(3), 185-189. http://doi.org/10.1016/j.wombi.2013.04.005

Moher, D., Liberati, A., Tetzlaff, J., Altman, D. G., \& Altman, D. (2009). Preferred Reporting Items for Systematic Reviews and Meta-Analyses: The PRISMA Statement. PLoS Medicine, 6(7), e1000097. http://doi.org/10.1371/journal.pmed.1000097

Morelli, A. B., \& Scorsolini-Comin, F. (2016). Repercussões da morte do filho na dinâmica conjugal de casais religiosos. Temas em Psicologia, 24(2), 565-577. http://doi.org/10.9788/TP2016.2-10

Muza, J. C., Sousa, E. N., Arrais, A. R., \& Iaconelli, V. (2013). Quando a morte visita a maternidade: Atenção psicológica durante a perda perinatal. Psicologia: Teoria e Prática, 15(3), 34-48. Disponível em: http://http://pepsic.bvsalud.org/pdf/ptp/v15n3/03.pdf

O'Leary, J. (2004). Grief and its impact on prenatal attachment in the subsequent pregnancy. Archives of Women's Mental Health 7, 7-18. https://doi.org/10.1007/s00737-0030037-1

Oishi, K. L. (2014). O Jardim de Julia: A vivência de uma mãe durante o luto. Psicologia: Teoria e Pesquisa, 30(1917), 5-11. https://doi.org/10.1590/S0102-37722014000100002

Oliveira, C. C. (2006). O luto pela criança que não nasceu. In R. M. M. Melamed \& J. Quayle (Orgs.), Psicologia em reprodução assistida: Experiências brasileiras. (pp. 207-225). Casa do Psicólogo.

Ouzzani, M., Hammady, H., Fedorowicz, Z., \& Elmagarmid. A. (2016). Rayyan - a web and mobile app for systematic reviews. Systematic Reviews, 5(210). http://doi.org/10.1186/s13643-016-0384-4

Paris, G. F., Montigny, F. de, \& Pelloso, S. M. (2016). Fatores associados ao estado de luto 
após óbito fetal: Estudo comparativo entre brasileiras e canadenses. Revista da Escola de Enfermagem da USP, 50(4), 546-553. http://doi.org/10.1590/S0080623420160000500002

Price, S. K. (2008). Stepping back to gain perspective: Pregnancy loss history, depression, and parenting capacity in the Early Childhood Longitudinal Study, Birth Cohort (ECLSB). Death Studies, 32(2), 97-122. http://doi.org/10.1080/07481180701801170

Rios, T. S., Santos, C. S. S., \& Dell'Aglio, D. D. (2016). Elaboração do processo de luto após uma perda fetal: Relato de experiência. Revista de Psicologia da IMED, 8(1), 98-107. https://doi.org/10.18256/2175-5027/psico-imed.v8n1p98-107

Sampaio, R. F., \& Mancini, M. C. (2007). Estudos de revisão sistemática: Um guia para síntese criteriosa da evidência científica. Revista Brasileira de Fisioterapia, 11(1), 83-89. https://doi.org/10.1590/S1413-35552007000100013

Sandler, I. N., Wolchik, S. A., \& Ayers, T. S. (2007). Resilience rather than recovery: A contextual framework on adaptation following bereavement. Death Studies, 32(1), 59-73. http://doi.org/10.1080/07481180701741343

Santos, A. L. D., Rosenburg, C. P., \& Buralli, K. O. (2004). Histórias de perdas fetais contadas por mulheres: Estudo de análise qualitativa. Revista de Saúde Pública, 38(2), 268-276. https://doi.org/10.1590/S0034-89102004000200017

Serafim, T. C., Camilo, B. H. N., Carizani, M. R., Gervasio, M. D. G., Carlos, D. M., Salim, N. R. (2021). Atenção à mulher em situação de óbito fetal intrauterino: Vivências de profissionais da saúde. Revista Gaúcha de Enfermagem, 42, e20200249. https://doi.org/10.1590/1983-1447.2021.20200249

Sijpt, E. van der. (2010). Marginal matters: Pregnancy loss as a social event. Social Science and Medicine, 71(10), 1773-1779. http://doi.org/10.1016/j.socscimed.2010.03.055

Silva, D. R. (2009). Famílias e situações de luto. In L. C. Osório \& M. E. P. do Valle (Orgs.), Manual de Terapia Familiar (pp. 376-398). Artmed.

Sugiura-Ogasawara, M., Suzuki, S., Ozaki, Y., Katano, K., Suzumori, N., \& Kitaori, T. (2013). Frequency of recurrent spontaneous abortion and its influence on further marital relationship and illness: The Okazaki Cohort Study in Japan. Journal of Obstetrics and Gynaecology Research, 39(1), 126-131. http://doi.org/10.1111/j.14470756.2012.01973.x

Üstündağ - Budak, A. M., Larkin, M., Harris, G., \& Blissett, J. (2015). Mothers' accounts of their stillbirth experiences and of their subsequent relationships with their living infant: An interpretative phenomenological analysis. BMC Pregnancy and Childbirth, 15(263). http://doi.org/10.1186/s12884-015-0700-3

Vidal, M. (2010). Gravidez após morte perinatal: Sobre a relação da mãe com o bebê sobrevivente. Ciencia \& Saúde Coletiva, 15(2), 3185-3190. https://doi.org/10.1590/S1413-81232010000800023

Walsh, F., \& McGoldrick, M. (2013). Bereavement: A family life cycle perspective. Family Science, 4(1), 20-27. http://doi.org/10.1080/19424620.2013.819228

Worden, J. W. (2013). Aconselhamento do luto e terapia do luto: Um manual para profissionais da saúde mental. Roca.

Submetido: $14 / 11 / 2017$

Reformulado: 03/05/2021

Aceito: 05/05/2021 


\section{Sobre os autores:}

Gabriela Vescovi é doutoranda em Psicologia pela Universidade Federal do Rio Grande do Sul.

Flávia Santos da Silva é mestranda em Psicologia e Saúde pela Universidade Federal de Ciências da Saúde de Porto Alegre (UFCSPA).

Daniela Centenaro Levandowski é doutora em Psicologia pela Universidade Federal do Rio Grande do Sul e docente dos Programas de Pós-graduação em Psicologia e Saúde e em Ciências da Saúde da Universidade Federal de Ciências da Saúde de Porto Alegre (UFCSPA).

Crístofer Batista da Costa é doutor em Psicologia Clínica pela Universidade do Vale do Rio dos Sinos (UNISINOS) e docente nas Faculdades Integradas de Taquara (FACCAT) e Faculdade do Centro de Estudos da Família e do Indivíduo (FACEFI).

Correspondência: gabriela.vescovi@gmail.com 